\title{
Numerical Cavitation Noise Prediction of a Benchmark Research Vessel Propeller
}

\author{
Savas Sezen ${ }^{1}$, Mehmet Atlar ${ }^{1}$, Patrick Fitzsimmons ${ }^{1}$, Noriyuki Sasaki ${ }^{1}$ \\ Giorgio Tani², Naz Yilmaz ${ }^{3}$, Batuhan Aktas ${ }^{4}$ \\ ${ }^{1}$ University of Strathclyde, Glasgow, UK \\ ${ }^{2}$ University of Genova, Genova, Italy \\ ${ }^{3}$ Technical University of Bursa, Bursa, Turkey \\ ${ }^{4}$ Fugro Structural Monitoring, Glasgow, UK
}

This paper presents the preliminary results of a numerical study for noise prediction of a benchmark propeller in open water/uniform flow conditions. The experimental benchmark test data for the research vessel, "The Princess Royal", were used for validation purposes. The numerical analyses were implemented by using a viscous solver based on the finite volume method while the experimental data were obtained from model tests conducted at the Genova University Cavitation Tunnel. The main aim of the study is to predict propeller hydro-acoustic performance under cavitating conditions. The hydrodynamic flow field was solved using a RANS (Reynolds-averaged NavierStokes) solver. The Schnerr-Sauer cavitation model based on a reduced Rayleigh-Plesset equation together with a VOF approach was used to model sheet cavitation on the propeller blades. The computed hydrodynamic characteristics and sheet cavity patterns were shown to be in good agreement with the Genoa experimental data, thus providing a firm basis for cavitating noise predictions. The hydro-acoustic performance of the model propeller was predicted by using a hybrid method. In the noise simulations, RANS equations were equipped with a porous FW-H (Ffowcs Williams-Hawkings) formulation. The different propeller operational conditions were simulated using this hybrid method. The numerical results were also validated with the experimental data for the propeller hydro-acoustic performance. Whereas such validations showed promising results by means of overall noise spectrum with the benchmark test cases in the low-frequency range, the numerical prediction overestimated the $1^{\text {st }}$ BPF values (around $20 \mathrm{~dB}$ ) in five loading conditions. Besides, in some loading conditions, especially between $200-800 \mathrm{~Hz}$, the difference between numerical predictions and the experiment was found around 5-10 $\mathrm{dB}$.

Keywords: CFD; Cavitation; FW-H; The Princess Royal research vessel; Propeller Underwater Radiated Noise (URN); RANS. 


\section{Introduction}

Cavitation is a complex fluid mechanics phenomenon that occurs due to the local static pressure drop and takes place in flowing liquids such as pumps, nozzles, injectors, turbines, propellers, and a variety of other fluid machinery components. It can lead to undesirable effects for marine propellers such as efficiency loss, hull/shaft vibrations, underwater radiated noise and blade erosion (Carlton, 2007). In the presence of the cavitation, the pressure amplitudes increase dramatically leading to the higher noise levels both in low and high-frequency regions.

Depending on the propeller operating conditions, the marine propeller can experience different types of cavitation such as sheet, bubble or cloud, tip, and hub vortex. Marine propeller cavitation is generally classified in two parts: cavitation attached to blade surfaces; and cavitation of the blade surfaces (e.g. tip vortex cavitation) (Oshima, 1994). Although the different cavitation types trigger the underwater propeller radiated noise considerably, the most common and often unavoidable is tip vortex cavitation, especially if the bursting or collapsing phenomenon occurs which is usually caused by effects of non-uniform flow (Konno et al, 2002). Underwater radiated propeller cavitation noise consists of tonal blade rate noise and broadband noise. While the tip vortex cavitation is a significant contributor to the broadband noise at high-frequency and medium low-frequency regions, sheet cavitation increases the noise levels in the low-frequency region (i.e. tonal blade rates) as well as the broadband region. It should be noted that in the presence of the collapsing phenomenon, tonal noise levels also increase in the noise spectrum.

Underwater Radiated Noise (URN) from ships has received increased attention over the last decade, in the design of improved naval systems, fishing vessels and increased comfort in cruise vessels, but primarily due to concern for the possible impact on marine wildlife. The issue was put on the political agenda of the Marine Strategy Framework Directive, MSFD, D11 2017/848 for a relevant qualitative descriptor for a good environmental status in the marine environment by the European Commission in 2016. Shipping noise originates from various sources on board a vessel. At low ship speeds, onboard machinery noise is dominant until propeller cavitation inception occurs, thus dominating the overall radiated noise spectrum. While it is not possible to avoid cavitation at service speed conditions for efficient commercial ships, various full-scale URN measurements have shown room for improvement. Among the same type of vessels, full-scale measurements have reported up to $20 \mathrm{~dB}$ difference in the measured noise levels. It may suggest that current practices in ship design can be improved scrutinised in terms of the URN characteristics and hence may lead to minimising the impact on ever-increasing ambient noise levels in the world's oceans. To reduce the ambient noise levels in the oceans, IMO (International Maritime Organization) has published a non-mandatory 
guideline for commercial ships (IMO, 2014). The scope of this guideline is to give general advice related to mitigation of underwater radiated noise to designers, ship operators, and owners.

In order to provide a better understanding of underwater radiated noise measurements, a Joint Research Programme (JRP)10, named "Noise Measurements", was established with the Hydro Testing Alliance Network of Excellence (HTA-NoE), completing its mission successfully in 2011 (AMT'11, 2011). The members of this group further decided to continue the URN investigations under the activities of a new working group entitled "Noise Community of Practice (Noise Cop) of the Hydro Testing Forum (HTF). A round-robin (RR) test programme was organized for prediction of open water cavitation and hydro-acoustic performance of the Newcastle University`s (UNEW) Deep-V type catamaran research vessel, "The Princess Royal". In the scope of the RR test programme, different facilities (i.e. University of Genoa UNIGE, University of Strathclyde, NMRI, SSPA, KRISO, CNR-INM, and MARIN) developed techniques to model cavitation and noise prediction and presented their results through hydrodynamic and hydro-acoustic performance for the Princess Royal Propeller. A general view of the ongoing round robin test programme with the Princess Royal vessel propeller was presented in the study of Tani et al. (2019b). Included in this study, were details of the different facilities, experimental set-ups, and data analysis methods. Besides, comparative results of the cavitation patterns and noise characteristics of the test propeller were presented and discussed based on the measurement results from the different facilities. In the scope of their study, the results of uniform flow conditions were given, and further studies were recommended.

URN can be predicted in some of the following ways, including experimental methods in cavitation tunnels and depressurised towing tanks, semi-empirical formulations, and computational fluid dynamics (CFD) methods. With the development of computer technologies, CFD tools (viscous or potential based codes) are becoming more popular but yet it is considered to require more validation (Wijngaarden, 2005 and Aktas et al. 2016). The application of the porous FW-H approach is relatively new field in maritime problems. It is due to the vital contribution of the non-linear noise terms on propeller hydro-acoustic performance. The non-linearities are mainly represented by turbulence and vorticity. Ianniello et al. 2013 pioneered the necessity of a porous FW-H approach which was first adopted by Ffowcs Williams and Hawkings (1969) and proposed as a numerical solution of the FWH equation by Di Francescantonio (1997) for hydro-acoustic problems. The definition of porous data surface, grid structure \& time step adopted in hydro-acoustic simulations has been still discussed (Lidtke et al., 2019). Due to the incompressible assumption in the maritime problems, numerical noise predictions are conducted coupled with hydrodynamic solvers such as RANS, Detached Eddy

* Corresponding author e-mail: savas.sezen@strath.ac.uk 
Simulation (DES) Large Eddy Simulation (LES) and Boundary Element Method (BEM) as a hybrid approach (hydrodynamic solver+acoustic analogy). Besides, the advantage of hybrid methods (numerical solver+acoustic analogy) is not dependent on the hydrophone location, which may be limited in typical experimental configurations. Additionally, experimental pressure sensors may be affected adversely by the facility reverberations during the measurements, and the resulting combined structural and acoustic signal can be tricky to separate clearly (Felli et al. 2014).

In recent years, studies for the prediction of propeller radiated noise have been increasing rapidly by the development of the numerical methods and experimental facilities. Numerical results have been compared with both model scale measurements, and experimental sea-trial data and capabilities of the numerical techniques have still been discussed in several studies. Hallander et al., (2012) examined the propeller underwater radiated noise under non-cavitating and cavitating conditions by using the various hybrid methods (such as BEM and RANS coupled FWH) and semi-empirical approaches. The underwater radiated noise induced by the propeller was predicted behind the wake of an LNG ship. As a result of the study, the difference in broadband noise region predicted by the FWH approach was found around $25 \mathrm{~dB}$ with respect to sea trial. Additionally, the propeller underwater radiated noise predicted from receivers located in upstream showed better agreement with the measured data than the receivers located in the downstream due to the intrinsic capability of the RANS approach in the downstream region. Lafeber et al., (2015) predicted underwater cavitating propeller noise for three different cases (i.e. a cruise liner, a container vessel, and a catamaran) using both numerical and experimental methods in the scope of the SONIC (Suppression of underwater Noise Induced by Cavitation) project. The experiments were conducted in MARIN`s Depressurized Wave Basin while the numerical tip vortex and sheet cavitation noises were predicted by using different tools such as tip vortex noise model (ETV) and sheet cavitation noise model with Brown semi-empirical formulation. It was found that ETV underpredicts the noise levels but presents a reasonable prediction in the noise spectrum. In contrast, Brown`s semi-empirical formulation provides a better estimation for the noise levels above $30 \mathrm{~Hz}$, except for lower frequencies, in comparison to model scale measurements. Kowalczyk and Felicjancik (2016) studied the hydroacoustic performance of marine propeller in non-uniform flow conditions by conducting experiments and numerical calculations. In this study, the flow field around the propeller blades was solved by RANS, while pressure fluctuations were calculated as a hydrodynamic pressure without using the acoustic analogy. The resulting pressure fluctuations were considered as acoustic pressures and noise levels were calculated at four-receiver locations. Sheet cavity patterns and noise levels up to $100 \mathrm{~Hz}$ were compared with the experimental results and good correlation was observed. Due to the grid

* Corresponding author e-mail: savas.sezen@strath.ac.uk 
resolution and insufficient tip vortex cavitation, the difference between numerical and experimental results by means of underwater radiated noise was found around 20-30 dB in higher frequencies. Bensow and Liefvendahl (2016) presented a methodology for prediction of propeller radiated noise under non-cavitating, cavitating and operating in behind hull conditions. The hybrid method, which is LES with porous FWH acoustic analogy, was adopted in the numerical calculations. The Research Vessel Princess Royal and her propeller were used in order to predict the underwater radiated noise at two different loading conditions, and the results were compared with the measurements. The numerical results showed that especially up to $400 \mathrm{~Hz}$, the difference in noise levels was found around $20 \mathrm{~dB}$ between numerical calculations and experiments. Sakamoto and Kamiirisa, (2018) investigated near-field cavitating propeller noise using a viscous CFD solver (DES) and Brown`s semi-empirical formulation in the near field in the model and full scale. In their study, four different propellers for existing ships were of the interest, and sheet cavitation was modeled by simulating the full-scale ship wake. Time series pressure fluctuations were captured to predict the propeller tonal noise levels, while the cavitation area was calculated using a viscous solver and used as input for Brown`s method to predict the propeller broadband noise. The results of the study showed that Brown`s formulation gives promising results to detect the upper bound limit of the broadband noise in comparison to experimental results. Li et al. (2018) investigated the full-scale chemical vessel`s hydro-acoustic performance using DES with a porous $\mathrm{FW}-\mathrm{H}$ approach. Numerical cavitating propeller noise predictions were compared with the sea trial data up to $1000 \mathrm{~Hz}$. It was believed that due to the insufficient tip vortex cavitation extension in numerical simulations, the noise level between $50-112 \mathrm{~Hz}$ where tip vortex cavitation is expected to be more dominant in full scale was underpredicted around $28 \mathrm{~dB}$ in numerical calculations in comparison to experiment. Above $200 \mathrm{~Hz}$, the numerical method significantly underpredicted noise levels with respect to sea trial data.

In a recent study Sezen and Kinaci (2019), model scale propeller hydro-acoustic performance was predicted by using RANS with the FW-H approach. In this investigation, the pressures which were gathered directly from a hydrodynamic solver, and the acoustic analogy were compared in the near field under non-cavitating and cavitating conditions. The results showed that the RANS based predicted pressures should not be extrapolated to the far-field by using the International Towing Tank Conference (ITTC) distance normalization. The FW-H solution is needed to predict the near field noise under cavitating conditions. In another study of Cianferra et al. (2019), a hybrid methodology was presented to predict the propeller radiated noise in open water conditions for a benchmark propeller, SVA-VP1304. The hydrodynamic field was solved by LES, and hydro-acoustic performance was predicted with the advective form of the FW-H formulation. The effects of different

* Corresponding author e-mail: savas.sezen@strath.ac.uk 
noise terms (i.e. thickness, loading, and quadrupole) were examined at different hydrophone locations. In a very recent study Lidtke et al. (2019), the hydro-acoustic performance of the INSEAN E779A model propeller were presented in a wakefield. RANS method was coupled with the porous FW-H approach. The effects of significant simulation parameters such as time step and grid resolution were investigated comprehensively. The results showed that the porous data surface should be selected carefully in order to avoid signal corruption due to the vortex penetrating with the surfaces. Despite the recent experimental and numerical studies in the field of a URN, reviewed above, the numerical prediction methods for the propeller URN still require more validation studies to predict the noise levels accurately, especially in the presence of the cavitation. The main aim of this study, therefore, is to present the preliminary numerical results for the benchmark Princess Royal model propeller. It was achieved by using the RANS method in combination with porous FW-H formulation in the low-frequency region. Besides, the numerical results were validated with the experiments under cavitating conditions.

In Section 2, the paper first presents details of the experimental set-up and test conditions of the benchmark propeller. The numerical models used in the CFD solver is given in Section 3. Verification and validation studies for the numerical solver are presented in Section 4. It is followed by the presentation and discussion of the numerical results in Section 5. Finally, in Section 6, concluding remarks are given, including suggestions for future work.

\section{Description of Experimental Set-up and Test Conditions}

The cavitating flow experiments and noise measurements were performed at the Cavitation Tunnel of the University of Genoa. This tunnel is a Kempf \& Remmers closed water circuit tunnel, schematically presented in Figure 1 . The tunnel has a 0.57 x $0.57 \mathrm{~m}$ square test section, and the total length of the test section is $2 \mathrm{~m}$. The maximum tunnel flow speed is $8.5 \mathrm{~m} / \mathrm{s}$ in the test section, and the nozzle contradiction ratio is 4:6:1 (Tani et al. 2017 and Tani et al. 2019a).

* Corresponding author e-mail: savas.sezen@strath.ac.uk 


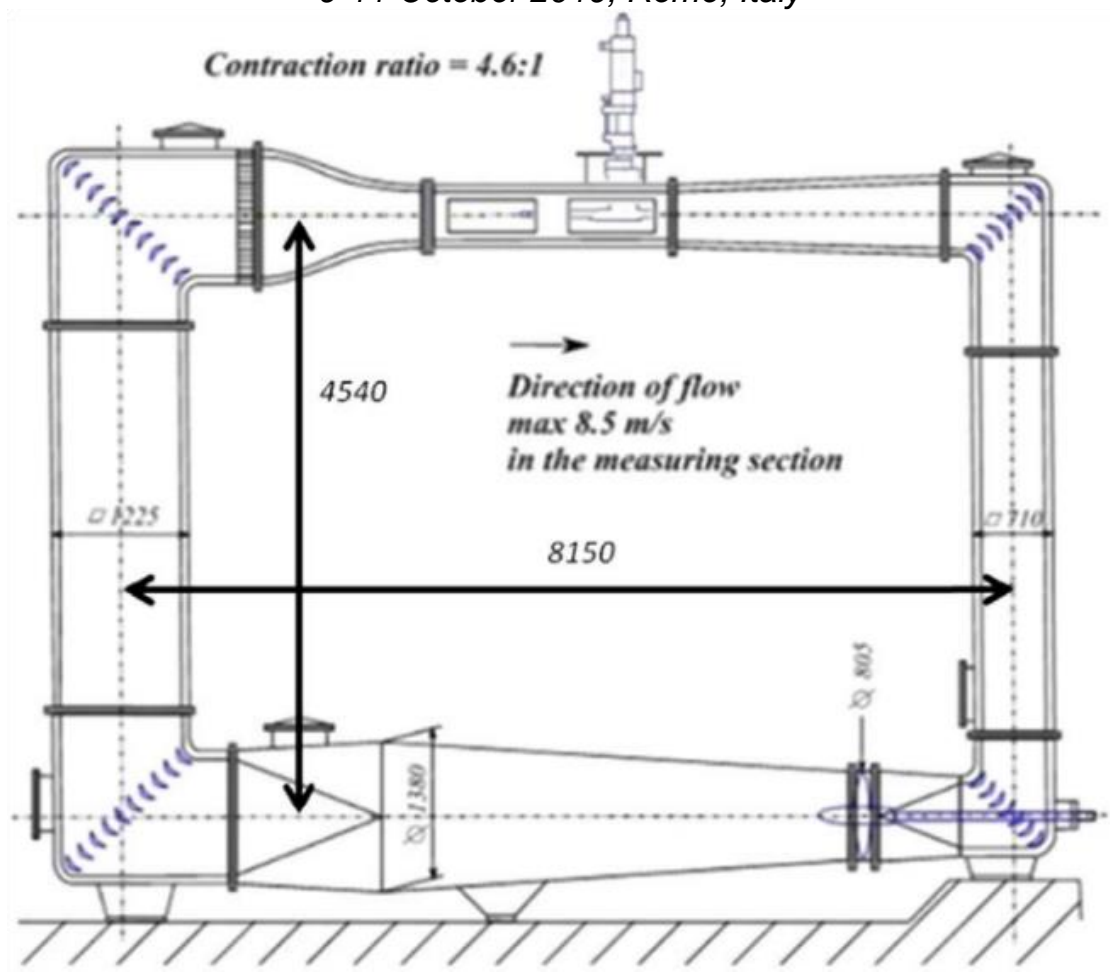

Figure 1: UNIGE Cavitation Tunnel (Tani et. al 2017).

A Kempf \& Remmers H39 dynamometer is used to measure the propeller thrust, torque and propeller rotational rate in the tunnel. Cavitation on the propeller blades is observed by using a mobile stroboscopic system. Additionally, three Allied Vision Tech Marlin F145B2 Firewire Cameras, with a resolution of 1392 x 1040 pixels and a frame rate up to $10 \mathrm{fps}$, are used for the cavitation observation. During the measurements, the quality of the water in the tunnel was checked regularly following the ITTC guidelines.

The URN measurements of the model propellers were conducted using three hydrophones (i.e. Reson TC4014 and two Bruel Kjaer type 8103) at different locations. Bruel \& Kjaer Type 2635 charge amplifiers were connected to hydrophones. The location of the hydrophones is shown in Figure 2. 
View from portside

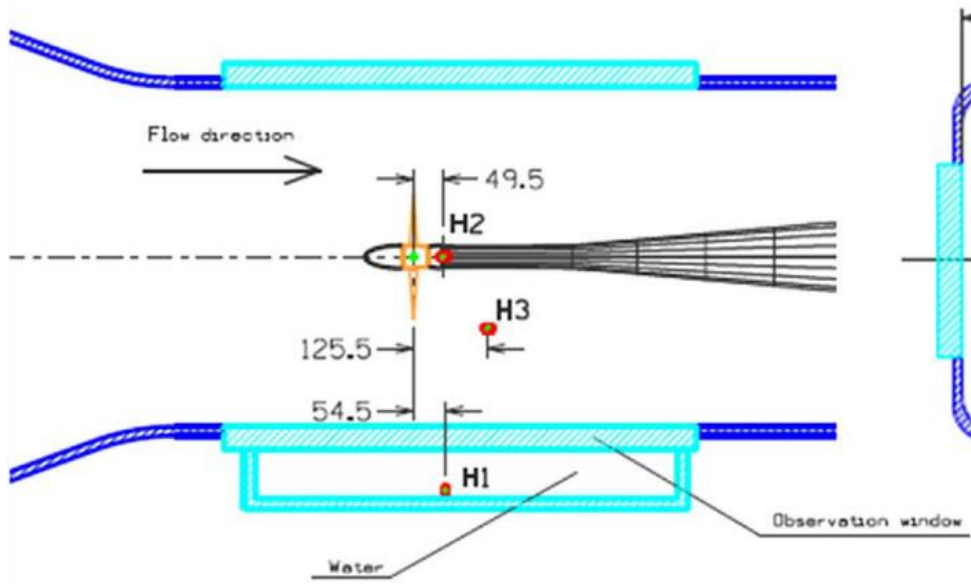

View from upstream

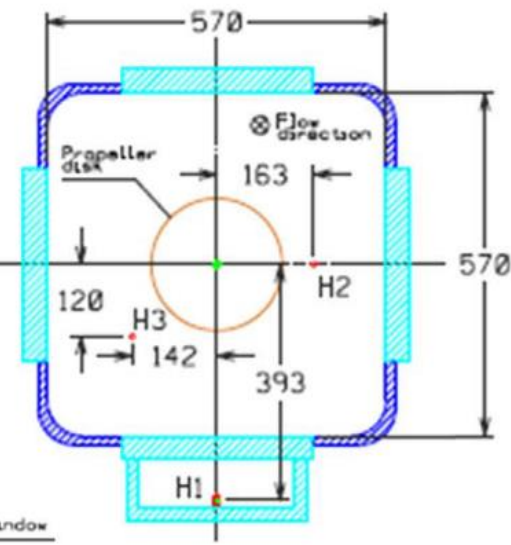

Figure 2. The hydrophones location in UNIGE Cavitation Tunnel (Tani et al. 2017).

Figure 2 shows hydrophone (H1) is placed in an acoustic chamber consisting of a small tank made of PMMA (Plexiglas), positioned on the observation window below the propeller and filled with water. The remaining hydrophones ( $\mathrm{H} 2$ and $\mathrm{H} 3)$ are placed inside the test section both downstream and outside the propeller slipstream. In the noise measurements, $2^{21}$ samples records were acquired with a $200 \mathrm{kHz}$ sampling frequency. The propeller operational conditions were identified according to the thrust coefficient and cavitation number identity. In the measurements, by taking the limits of the cavitation tunnel, the propeller rotational rate was selected as high as maximum to set to maximum Reynolds number. The flow speed was adjusted in the cavitation tunnel in accordance with the given propeller rotational rate to obtain the required thrust coefficient.

The cavitation number is generally derived with respect to propeller rotational rate by using Equation 1 ;

$$
\sigma_{N}=\frac{P_{0}-P_{V}}{\frac{1}{2} \rho(n D)^{2}}
$$

where $P_{0}$ is the static pressure, $P_{0}=P_{a t m}+\rho g h,(\mathrm{~Pa}) ; \rho$ is the density of water, $\left(\mathrm{kg} / \mathrm{m}^{3}\right) ; \mathrm{g}$ is the acceleration of gravity, $\left(\mathrm{m} / \mathrm{s}^{2}\right) ; \mathrm{h}$ is the propeller shaft immersion, $(\mathrm{m}) ; P_{V}$ is the vapour pressure, $(\mathrm{Pa}) ; \mathrm{n}$ is the propeller rotational rate, $(\mathrm{rps})$ and $\mathrm{D}$ is the propeller diameter, $(\mathrm{m})$. Propeller thrust $\left(K_{T}\right)$, torque $\left(K_{Q}\right)$ coefficients and efficiency $\left(\eta_{0}\right)$ values are calculated for different advance coefficients. The advance ratio is defined in Equation 2;

$J=\frac{V_{A}}{n D}$

Thrust, torque coefficients, and efficiency value are calculated as follows, respectively, 
$K_{T}=\frac{T}{\rho n^{2} D^{4}}$

$K_{Q}=\frac{Q}{\rho n^{2} D^{5}}$

$\eta_{0}=\frac{J}{2 \pi} \frac{K_{T}}{K_{Q}}$

where $\mathrm{T}$ is the propeller thrust, $(\mathrm{N})$; $\mathrm{Q}$ is the propeller torque, $(\mathrm{Nm})$; and $\mathrm{V}_{\mathrm{A}}$ is the propeller advance speed (this is ground speed or incoming flow at infinity upstream) $(\mathrm{m} / \mathrm{s})$. Reynolds number can be calculated for the marine propeller as follows;

$R e=\frac{n D^{2}}{v}$

where $v$ is the kinematic viscosity, $\left(\mathrm{m}^{2} / \mathrm{s}\right)$.

Within the scope of the round-robin test campaign, the model propeller of The Princess Royal was selected to predict the propeller underwater radiated noise, since the information was available for the full-scale ship together with sea trials. Figure 3 and Table 1 show the geometry and its main particulars.

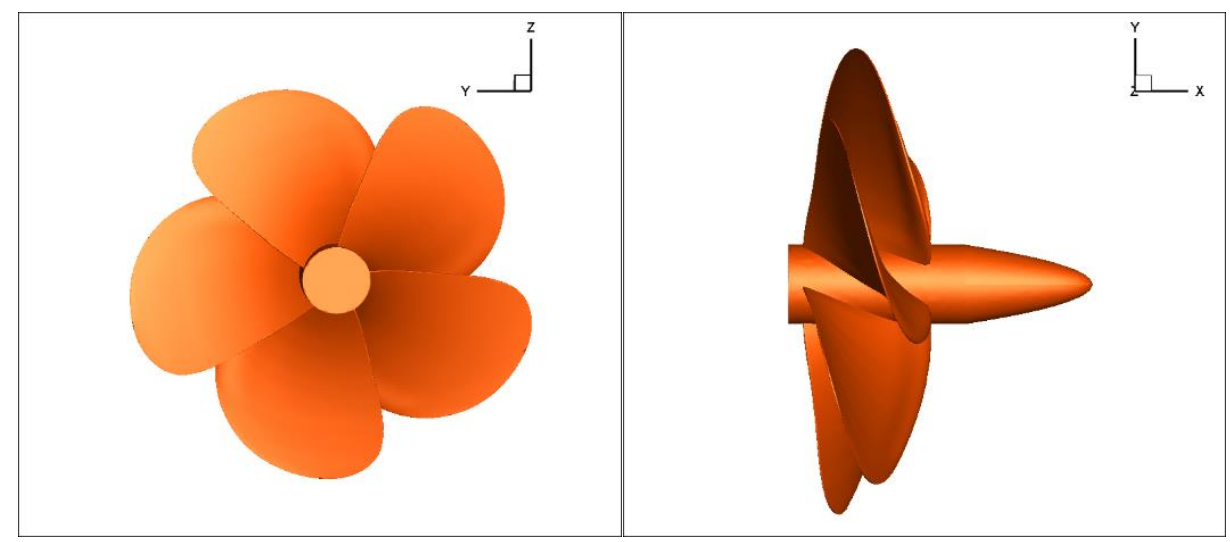

Figure 3. Model scale of Princess Royal propeller. 
Table 1: Model propeller main characteristics.

\begin{tabular}{|l|l|}
\hline Propeller Diameter $(\mathrm{m})$ & 0.22 \\
\hline Scale ratio $(\lambda)$ & 3.41 \\
\hline Number of Blades, $\mathrm{Z}$ & 5 \\
\hline P/D at $0.7 \mathrm{R}$ & 0.8475 \\
\hline Expanded Blade Area Ratio & 1.057 \\
\hline $\begin{array}{l}\text { Hub to Propeller Diameter Ratio } \\
\left(\mathrm{D}_{\mathrm{H}} / \mathrm{D}\right)\end{array}$ & 0.18 \\
\hline Rake Angle $\left({ }^{0}\right)$ & 0 \\
\hline Skew Angle $\left(^{(}\right)$ & 19 \\
\hline Propeller Type & Fixed Pitch Propeller \\
\hline Blade Loading Distribution & NACA a=0.8 \\
\hline Thickness Distribution & NACA 66 modified \\
\hline
\end{tabular}

During the round-robin test campaign, the propeller URN was predicted for both uniform and inclined flow conditions. However, in the scope of this study, experimental and numerical results were presented only for uniform flow conditions. The test matrix for uniform flow is listed in Table 2.

Table 2: Operational conditions used in the experiments and CFD analysis.

\begin{tabular}{|c|c|c|c|}
\hline Loading Condition & $\mathrm{J}$ & $\sigma_{N}$ & $\mathrm{n}(\mathrm{rps})$ \\
\hline $\mathrm{C} 1$ & \multirow{3}{*}{0.4} & 2.22 & \multirow{6}{*}{35} \\
\hline $\mathrm{C} 2$ & & 1.30 & \\
\hline $\mathrm{C} 3$ & & 0.72 & \\
\hline $\mathrm{C} 4$ & \multirow{3}{*}{0.5} & 3.48 & \\
\hline $\mathrm{C} 5$ & & 2.03 & \\
\hline C6 & & 1.13 & \\
\hline
\end{tabular}

\section{CFD Methodology}

In this section, details are given of the numerical models, computational domain \& grid structure used to solve flow around non-cavitating and cavitating propeller with an acoustic analogy.

\subsection{Computational Domain \& Mesh Generation}

Figure 4 provides a general overview of the computational domain used in the hydrodynamic and hydro-acoustic simulations. A cylindrical computational domain comprises the three regions, for noise calculations, namely static, rotating and porous surface regions. The upstream and downstream extents of the domain were set 4.5D and 10.5D, respectively, with the domain radius of $4 \mathrm{D}$. This means that computation domain dimensions were extended with respect to the experimental set-up. Propeller blades and hub were defined as a no-slip wall to satisfy the kinematic boundary condition. 


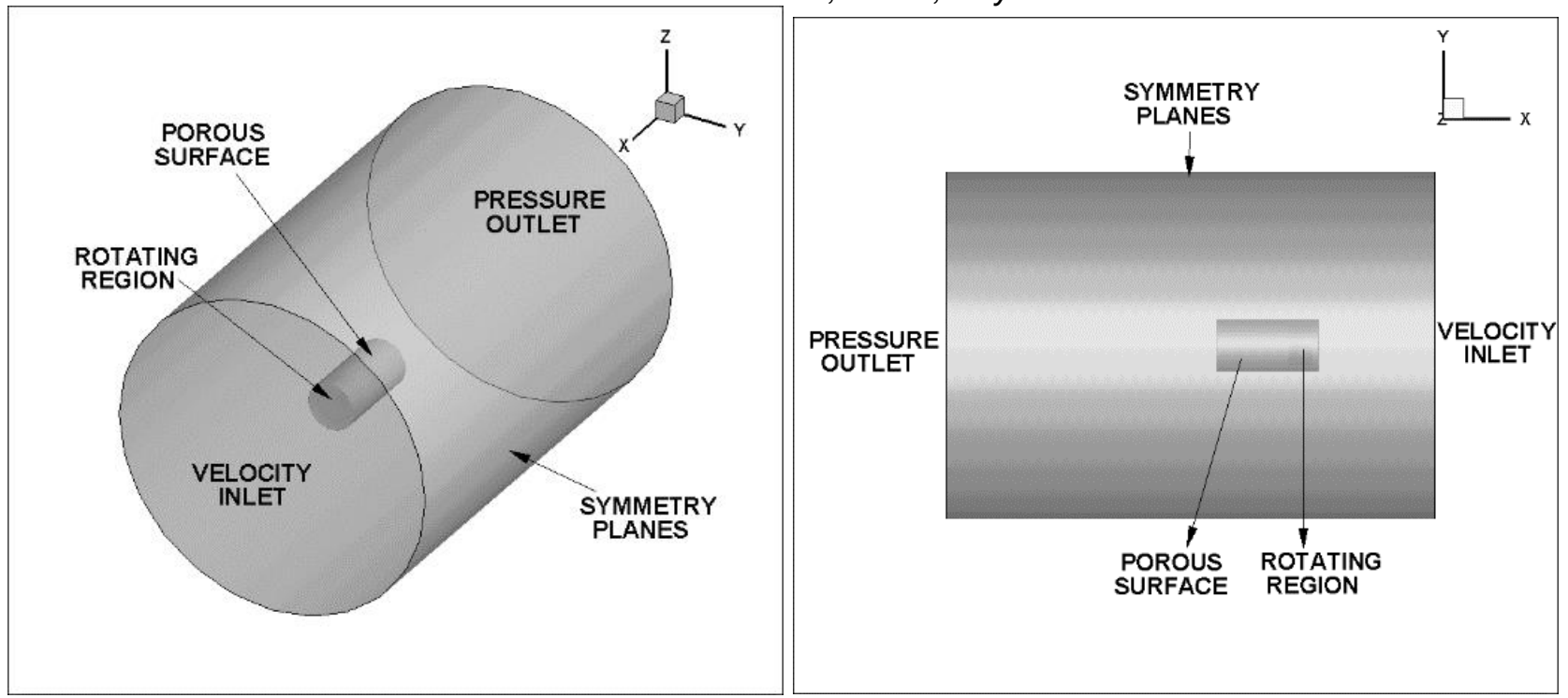

Figure 4. Computational domain and boundary conditions.

The Porous Data Surface (PDS) was situated outside of the interface between the static and rotating regions. The optimum location and size of the PDS, which encompasses all the non-linear effects, should be selected carefully to minimize any spurious effects (Lidtke et al. 2019). A view of the PDS and dimensions are given in Figure 5.

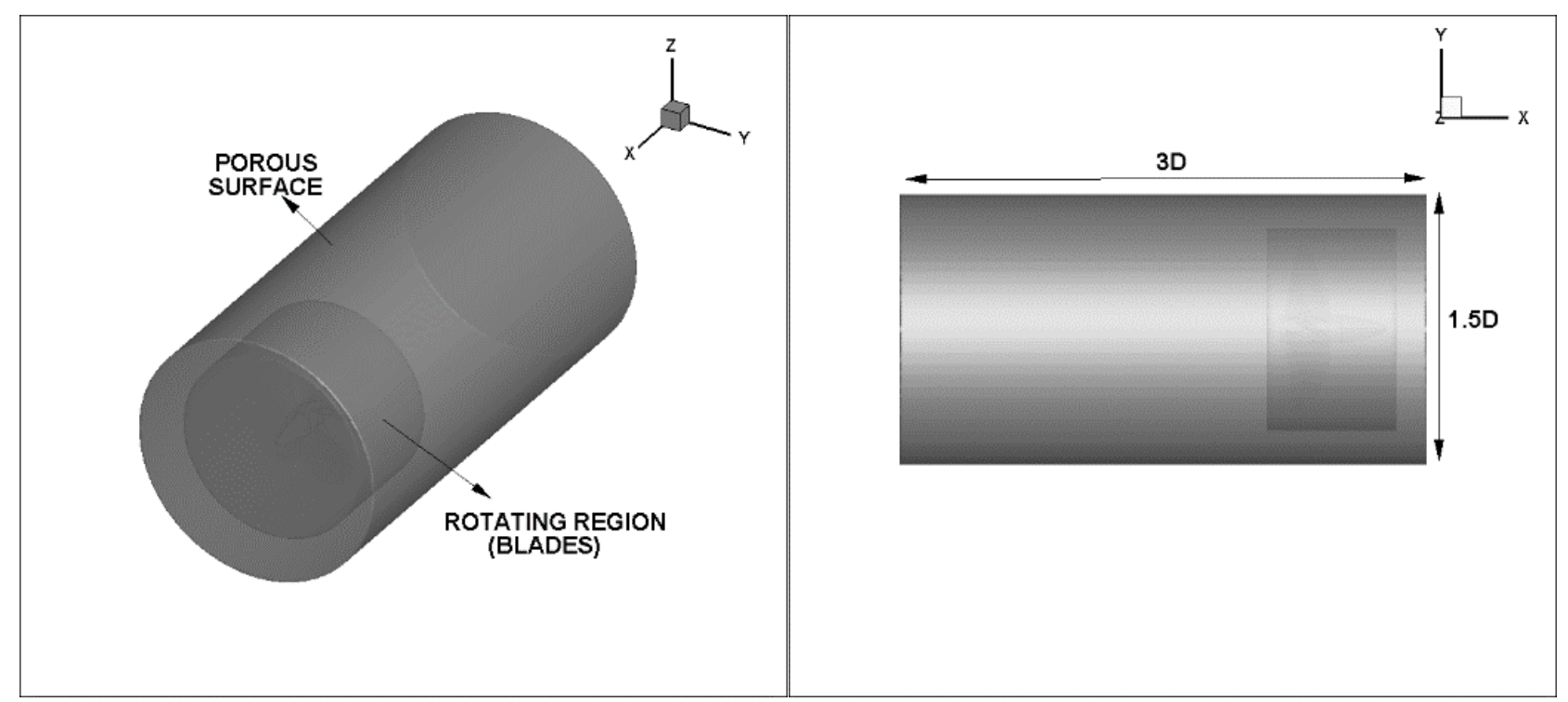

Figure 5. Side view of the PDS and its dimensions.

An unstructured grid structure was used in Figure 6 to discretise the computational domain in compliance with the Finite Volume Method (FVM). Additional volumetric control regions were applied around the propeller blades and in the near field to capture the flow field precisely.

In the hydro-acoustic simulations, the grid structure around the propeller blades and in the near field region was changed in comparison to the hydrodynamic simulations. It is since acoustic pressures are considered more sensitive to grid refinement than hydrodynamic inputs which are obtained from the 
hydrodynamic solver (Lloyd et al., 2015). The average wall y+ value in hydro-aoustic simulations was calculated around 120, which is suitable for $\mathrm{k}-\omega \mathrm{SST}$ turbulence modelling with all $\mathrm{y}+$ treatment method in Star CCM+, 2018.

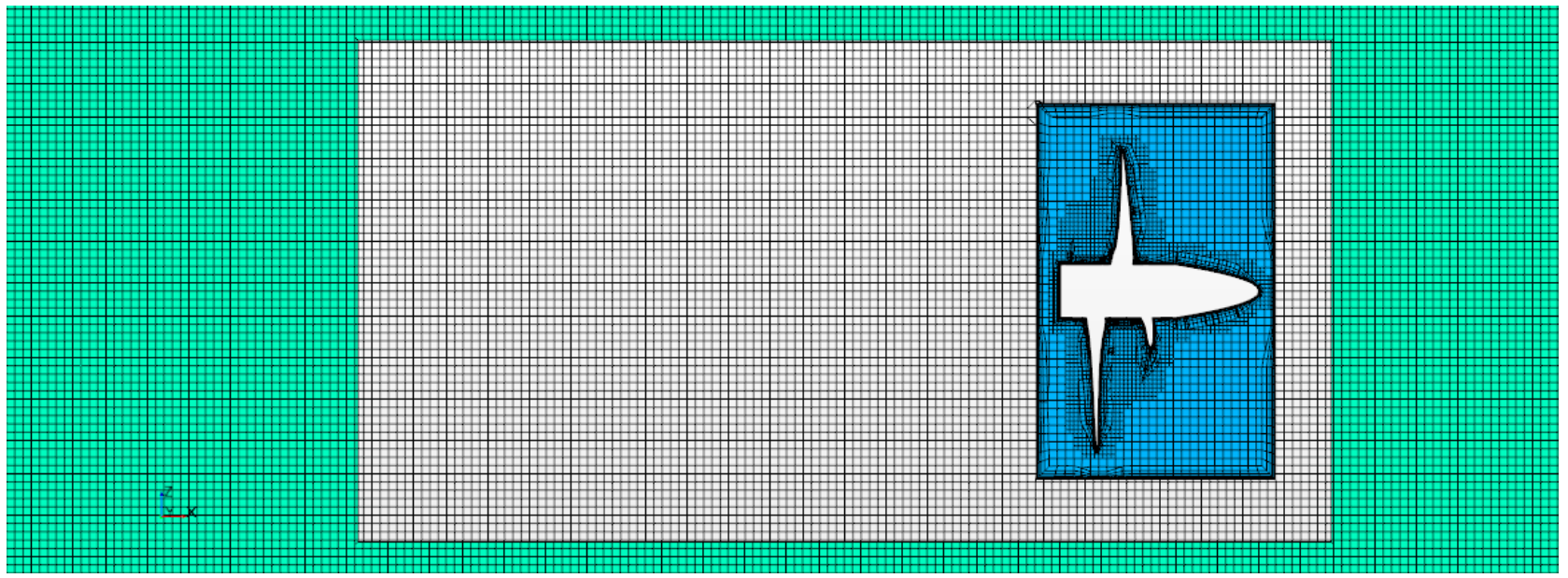

Figure 6. Unstructured grid distributions on the computational domain.

\subsection{Hydrodynamic Model}

The governing flow equations were solved using the Star-CCM+ commercial CFD code that has been widely used to predict propeller hydrodynamic and hydro-acoustic performance (Star CCM+,2018). In this code, a segregated flow model was adopted to solve the steady and unsteady RANS equations with the k- $\omega$ SST turbulence model. In the numerical calculations, momentum equations were discretised using the second-order scheme. Temporal discretization was also done using a secondorder scheme. The internal iteration was set as 10 in the simulations. According to the International Towing Tank Conference (ITTC, 2014) recommendations, the time-step is calculated such that the propeller rotational rate between 0.5-2 degree of the propeller rotational rate. Therefore, the step was set as 1 degree of the propeller rotational rate in the simulations. Once hydrodynamic simulations converge, the hydro-acoustic data were collected at 6 degrees of the propeller rotational rates around 20 propeller rotational cycles.

Propeller rotational motion was simulated by Rigid Body Motion (RBM) and Moving Reference Frame (MRF) techniques. In the first step, MRF was adopted based on the steady solution of the governing equations. Although more realistic results can be obtained from the unsteady solution, due to the cost-savings, the propeller open water simulations can be generally conducted based on the steady solution. However, the RBM technique is preferred for unsteady cases such as cavitation and noise since it is considered to be the most accurate way to obtain the change of flow variables with time. At each time step, the governing equations are solved with a given grid position. In this study, 
the MRF technique was performed to compute non-cavitating propeller hydrodynamic performance, whereas the RBM was applied for the propeller URN simulations under cavitating conditions.

Cavitation on the propeller blades was modelled with the well-known Schnerr-Sauer cavitation model by using the Volume of Fluid (VOF) approach to elucidate the phase changing of liquid into vapour. This model is based on the reduced Rayleigh-Plesset equation and neglects the impact of bubble growth acceleration, viscous effects, and surface tension. The cavitation model is based on the homogenous distribution of bubble seeds throughout the liquid. In this model, the bubble growth rate is predicted using Equation 7;

$$
\left(\frac{d R}{d t}\right)^{2}=\frac{2}{3}\left(\frac{p_{s a t}-p_{\infty}}{\rho}\right)
$$

where, $p_{\infty}$ is the pressure of the liquid, $(\mathrm{Pa}) ; p_{\text {sat }}$ is the saturation pressure, $(\mathrm{Pa})$.

\section{3, Hydro-acoustic Model}

Hydro-acoustic simulations were carried out using the Ffowcs Williams-Hawkings (FWH) acoustic analogy based on Lighthill's equation (Lighthill, M.J., 1952). It is the re-arrangement form of the continuity and momentum equations into nonhomogeneous wave equations (Williams and Hawkings, 1969). The generalised FW-H equation is given in terms of generalised function in Equation 8;

$$
\begin{aligned}
D^{2} p(x, t)=\frac{\partial}{\partial t}\left\{\left[\rho_{0} v_{n}+\rho\left(u_{n}-v_{n}\right)\right] \delta(f)\right\}-\frac{\partial}{\partial x_{i}}\left\{\left[\Delta P_{i j} \hat{n}_{j}+\rho u_{i}\left(u_{n}-v_{n}\right)\right] \delta(f)\right\} \\
+\frac{\partial}{\partial x_{i} \partial x_{j}}\left\{T_{i j} H(f)\right\}
\end{aligned}
$$

Here, $p$ is the acoustic pressure disturbance, $\mathrm{Pa} ; \mathrm{D}^{2}$ is the $\mathrm{D}^{\prime}$ Alembert operator, $T_{i j}$ is Lighthill stress tensor, $\mathrm{N} ; u_{i}$ and $v_{i}$ is the fluid and surface velocity components, $\mathrm{m} / \mathrm{s}$ respectively. $\tilde{\rho}=\rho-\rho_{0}$ is the density perturbation, $\mathrm{kg} / \mathrm{m}^{3}$ with respect to the undisturbed medium and $\mathrm{n}$ indicates the projection along the outward normal to the surface. The first term on the right-hand side of Equation 8 represents the thickness (monopole) contribution, whereas the second term on the right-hand side is loading (dipole) contribution. The latter part on the right-hand side of the equation is the quadrupole (nonlinear) noise term. The first two terms are evaluated by solving the surface integrals, and the latter one is evaluated using volume integral. D'Alembert operator and $\mathrm{T}_{\mathrm{ij}}$ Lighthill Stress tensor can be written as follows;

$$
D^{2}=\frac{1}{c_{0}{ }^{2}} \frac{\partial^{2}}{\partial t^{2}}-\nabla^{2}
$$


$T_{i j}=\rho u_{i} u_{j}+P_{i j}-c_{0}^{2} \tilde{\rho} \delta_{i j}$

$c_{0}$ is the sound speed, $\mathrm{m} / \mathrm{s} ; P_{i j}$ represents the compressive stress tensor, $\mathrm{N}$ and $\Delta P_{i j}=P_{i j}-p_{0} \delta_{i j}$. ( $\delta_{i j}$ is the Kronecker operator). According to Williams and Hawkings` approach, the noise surface to define the sound source should be impermeable, and so they had generally considered an impermeable surface to be used. By doing this, the surface terms are comprised of linear components, namely thickness and loading. This form of the FWH is known as Farassat 1A, which is a proposed solution to the FW-H equation using Green Functions by Brentner and Farassat, 2003. This form of the FW$\mathrm{H}$ equation is widely used mainly in the aeroacoustic community. For maritime problems, the Farassat 1A solution loses its importance, and it becomes less desirable because of the contribution of nonlinear noise terms, particularly in the far-field.

In the numerical calculations, the integration surface covered the source region (in our case propeller), and it was placed far from the body where includes all possible non-linear noise sources (Lyrintzis, 2003). By doing this way, the volume integral term in the FW-H equation is transformed to surface integral term. In this study, the porous surface was composed of three parts; the entrance \& outer caps (located downstream and upstream) and the cylindrical lateral surface. The downstream cap of porous surface cuts the vortex distribution in the slipstream. Therefore, the porous acoustic analogy needs an endcap scheme to prevent potential spurious noise which may affect the overall noise signature induced by the propeller due to the truncation errors (Nitzkorski, 2015). Therefore, the open-ended surface was used in the simulations to prevent the vortex penetrating and spurious noise. Having said that there are several key features of a porous FWH surface such as the placement and location that are still not specified clearly and open to discussion, e.g. (Bensow and Liefvendahl, 2016, Li et al., 2018).

It should be noted that the linear terms of the FW-H equation lose their physical meaning and thus, the non-linear terms, which are located within the PDS region are accounted for solving thickness and loading noise terms (Ianniello et al. 2003 and Lloyd et al. 2014). Since PDS encompasses all of the noise sources, non-linear effects are generally ignored outside of the PDS region. The possible numerical solution of the FW-H (Equation 8) is obtained by defining the two acoustic variables (Equation 11 and 12) under the porous FW-H hypothesis and incompressible flow assumption.

$U_{i}=u_{i}$

$L_{i}=P_{i j} \hat{n}_{j}+\rho u_{i}\left(u_{n}-v_{n}\right)$ 
where $u$ and $v$ are the fluid and porous surface velocities. In the assumptions of neglecting of density fluctuations $\rho=\rho_{0}$ and that there is a stationary data surface $\left(v_{n}=0\right)$, the porous FW-H equation becomes,

$$
4 \pi p(x, t)=\int_{S}\left[\frac{\rho_{0} \dot{U}_{n}}{r}\right]_{\tau} d S+\int_{S}\left[\frac{\dot{L}_{r}}{c_{0} r}\right]_{\tau} d S \int_{S}\left[\frac{L_{r}}{r^{2}}\right]_{\tau} d S
$$

Here, $\mathrm{r}$ is the radiation direction, and dot defines a source time derivative with respect to retarded time. Subscripts $r$ and $n$ define the dot product of a quantity with a unit vector either radiation or normal directions, respectively.

\section{Verification and Validation of Hydrodynamic Model}

The verification study was conducted for non-cavitating open water conditions using the Grid Convergence Index (GCI) method based on Richardson, (1911) for extrapolation using three different grids (fine, medium and coarse). The uncertainty study was only applied for grid-spacing with respect to the defined methodology proposed by Celik et al. 2008. It was conducted for both thrust coefficient $\left(\mathrm{K}_{\mathrm{T}}\right)$ and torque coefficient $\left(10 \mathrm{~K}_{\mathrm{Q}}\right)$ at $\mathrm{J}=0.4$ using the refinement factor $\mathrm{r}=\sqrt{2}$ since it can be greater than 1.3. For the sake of completeness, this procedure is summarized;

The difference between the three different grids solutions should be determined by Equation 14 .

$\varepsilon_{21}=\varphi_{2}-\varphi_{1}, \quad \varepsilon_{32}=\varphi_{3}-\varphi_{2}$

Here, $\varphi_{1}, \varphi_{2}$ and $\varphi_{3}$ are fine, medium and coarse mesh grid solutions, respectively. The ratio of solution scalars is used to calculate the convergence condition by Equation 15

$$
R=\frac{\varepsilon_{21}}{\varepsilon_{32}}
$$

The solution type is identified in accordance with the value of R. Four different types of solutions are available (Stern et al., 2006). If the convergence condition $(\mathrm{R})$ is found between $0<\mathrm{R}<1$, the procedure is implemented according to the methodology of Celik et al. 2008. Apparent order (p) of the method is calculated by Equation 16.

$$
\begin{aligned}
& p=\frac{\operatorname{In}\left\|\frac{\varepsilon_{32}}{\varepsilon_{21}}+q\right\|}{\operatorname{In}\left(r_{21}\right)} \\
& q=\operatorname{In}\left(\frac{r_{21}-s}{r_{32}-s}\right)
\end{aligned}
$$


Here, $r_{21}$ and $r_{32}$ is the refinement factors which are calculated by $r_{21}=\sqrt[3]{\frac{N_{1}}{N_{2}}}$. ( $\mathrm{N}$ is the cell number).

$s=\operatorname{sgn}\left(\frac{\varepsilon_{32}}{\varepsilon_{21}}\right)$

The extrapolated value is:

$\varphi_{\text {ext }}^{21}=\frac{\left(r^{p} \varphi_{1}-\varphi_{2}\right)}{\left(r^{p}-1\right)}$

The approximate relative error and extrapolated relative error are:

$e_{a}^{21}=\left|\frac{\varphi_{1}-\varphi_{2}}{\varphi_{1}}\right| \quad e_{e x t}^{21}=\frac{\left|\varphi_{\text {ext }}{ }^{12}-\varphi_{1}\right|}{\varphi_{\text {ext }}{ }^{12}}$

Ultimately, the GCI index is calculated by:

$G C I_{\text {fine }}^{21}=\frac{1.25 e_{a}^{21}}{r_{21}^{p}-1}$

Table 3 gives element counts for the fine, medium and coarse grids, together with solution results and uncertainty values.

Table 3: Numerical Uncertainty for $\mathrm{K}_{\mathrm{T}}$ and $10 \mathrm{~K}_{\mathrm{Q}}$ at $\mathrm{J}=0.4$

\begin{tabular}{|c|c|c|c|c|c|}
\hline & $\varphi_{1}$ & $\varphi_{2}$ & $\varphi_{3}$ & $R$ & $\% G C I_{F I N E}$ \\
\hline Number of Cells & $2.9 \mathrm{M}$ & $1.1 \mathrm{M}$ & $0.49 \mathrm{M}$ & - & - \\
\hline $\mathrm{K}_{\mathrm{T}}$ & 0.2231 & 0.2227 & 0.2221 & 0.66 & 0.379 \\
\hline $10 \mathrm{~K}_{\mathrm{Q}}$ & 0.3448 & 0.3465 & 0.3488 & 0.72 & 1.257 \\
\hline
\end{tabular}

Based on the result of the verification study, the fine mesh was selected for the non-cavitating open water propeller simulations. Numerical and experimental results for different advance coefficients are given in Figure 7. In the hydrodynamic simulations, the propeller rotational rate was constant at $\mathrm{n}=20 \mathrm{rps}$ in compliance with the experiment. The same grid structure was not adopted for acoustic simulations since hydro-acoustic simulations are generally more sensitive to mesh quality than the traditional open water hydrodynamic simulations (e.g. determination of the open water variables such as $\mathrm{K}_{\mathrm{T}}, \mathrm{K}_{\mathrm{Q}}$ ). Therefore, suitable mesh structure has to be implemented to capture the pressure fluctuations in the near field of the propeller since the FW-H based analogy carries the pressure from near field to far-field as a transfer function (Lloyd et al. 2015). Therefore, the solution of the near field requires a finer grid structure in the vicinity of the propeller blades while the open water 
hydrodynamic characteristics can be calculated with a lesser number of element count in comparison to the cavitation and hydro-acoustic simulations. Having said that the grid structure to be adopted for the hydro-acoustic simulations should meet two main criteria: (i) the grid should be sufficient enough to resolve/model the sound generating turbulence scales, particularly in the propeller slipstream where the contributions due to the non-linear noise sources (i.e. turbulent and vorticity) are more dominant than the contribution due to the blade dynamics (i.e. blade thickness and loading); (ii) additionally, the grid structure should enable to resolve to the satisfactory propagation of the acoustic waves towards the locations of the receivers (Star CCM+, 2018).

Besides, the propeller rotational motion is generally modelled by a sliding interface approach (also known as Rigid Body Motion (RBM) approach) in numerical calculations. However, it is considered that the numerical or spurious noise, which is triggered by a sliding interface, may deteriorate the overall noise signature during computations (Lloyd et al. 2015), which in turn, may result in the lower or higher URN predictions compared to the experimental results. Therefore, any potential (additional) spurious noise prediction, which will pollute the overall acoustic pressure, can be avoided by preventing any mesh size changes inside the noise source region (Star CCM+, 2018). Besides the cells, which are inside the noise source region, were aligned with a sliding interface to facilitate the transition between two regions as well as to avoid numerical noise. Almost uniform cell size, particularly in $\mathrm{X}$ direction, was adopted to reduce the numerical diffusion. At least 20 cells per wavelength should be used to solve the shortest wavelengths in the simulations (Star CCM+, 2018). Having said that the difference of the mesh structures adopted in both hydrodynamic and hydroacoustic simulations was discussed in the 27th ITTC Conference (ITTC, 2014) and recommendations were made as follows: the grid resolution in space must be high enough and the quality of the mesh, such as aspect ratio, skewness, etc. should be adopted carefully. In this way the grid numbers in CFD simulations for hydrodynamic noise prediction are much more than those used for ship resistance or propeller thrust/torque computations, generally one or two orders of magnitude more. Meanwhile, the upper frequency of the fluctuating flow field (and fluctuating forces) is limited because of the mesh size and time step used". Additionally, to the best of the authors`knowledge, there is no specific uncertainty method conducted for hydro-acoustic simulations such as pressure fluctuations, overall 
noise signals etc. Hence, a grid of about $6.8 \mathrm{M}$ cells was used for the hydro-acoustic simulations and uncertainty study for the hydro-acoustic simulations was not performed within the scope of this study.

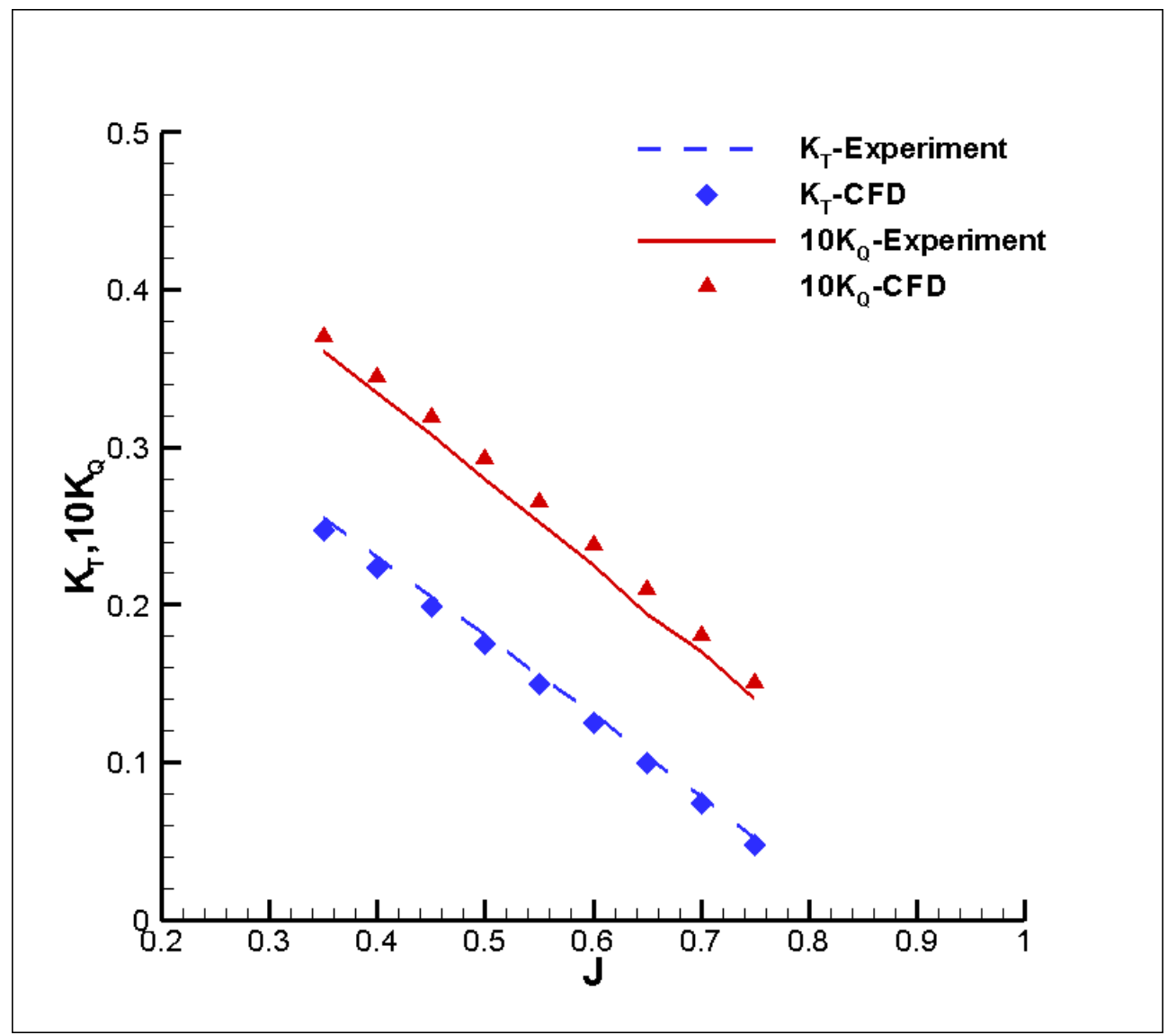

Figure 7. Comparison of thrust and torque coefficients.

\section{Results and Discussions}

Results and discussions for the cavitation observations, noise predictions and as well as propeller thrust coefficients are presented for the six different loading conditions, (C1 to C6) as shown in Table 2.

\subsection{Cavitation Observation}

Figure 8 and Figure 9 show the predicted and experimentally observed cavitation patterns on the propeller blades for $\mathrm{J}=0.4$ and $\mathrm{J}=0.5$, respectively. In the round-robin (RR) test campaign, sheet cavitation was observed slightly less extended towards inner radii than other facilities namely, SSPA, MARIN, and ECT, especially for the $\mathrm{C} 2$ and C3. This difference is considered due to the development of the boundary layer. During the experiments in UNIGE cavitation tunnel, a slightly lower propeller shaft rate (i.e. low Reynolds number) was used, and the portion of the laminar boundary layer could be present at inner radii. On the other hand, the numerical calculation is conducted in fully turbulent flow conditions, hence the slight overestimation of sheet cavitation in CFD computations might partially be because of this. 
As can be seen in these figures, the numerical results show a similar sheet cavitation pattern whilst tip vortex cavitation could not be observed as a grid refinement technique, such as Adaptive Mesh Refinement (AMR) was not implemented at the propeller blade tips (Yilmaz et al. 2019). Tip vortex cavitation in the slipstream can be observed accurately using different adaptive mesh refinement techniques and scaling resolving simulations such as LES and DES. Besides, the RANS approach can be considered to be a reliable model to observe the sheet cavitation on the propeller blades (Gaggero et al. 2014). In the scope of this study, the main aim was to predict the propeller underwater radiated noise in the low-frequency region due to the limitation of the RANS approach for broadband noise prediction. Moreover, the effects of tip vortex on propeller radiated noise might be stronger for the hydrophones located in the downstream region (Lloyd et al, 2015). Ultimately, observation of tip vortex cavitation, which is the main contributor to broadband noise, especially in the presence of such off-blade dynamic phenomena or bubble collapse, was not considered this study.

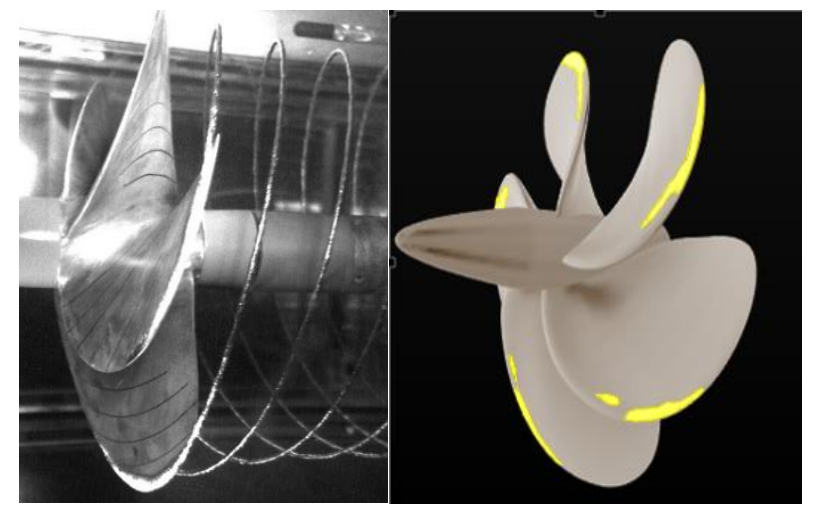

$\mathrm{C} 1, \mathrm{~J}=0.4, \sigma_{\mathrm{N}}=2.22\left(\mathrm{Left} ;\right.$ Experiment, Right; RANS $\left.\left(\alpha_{\mathrm{V}}=0.5\right)\right)$

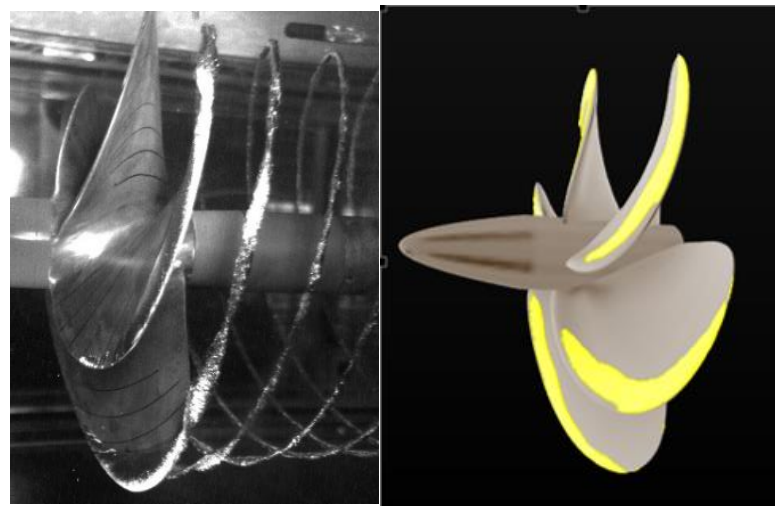

$\mathrm{C} 2, \mathrm{~J}=0.4, \sigma_{\mathrm{N}}=1.3\left(\mathrm{Left} ;\right.$ Experiment, Right; RANS $\left.\left(\alpha_{\mathrm{V}}=0.5\right)\right)$ 


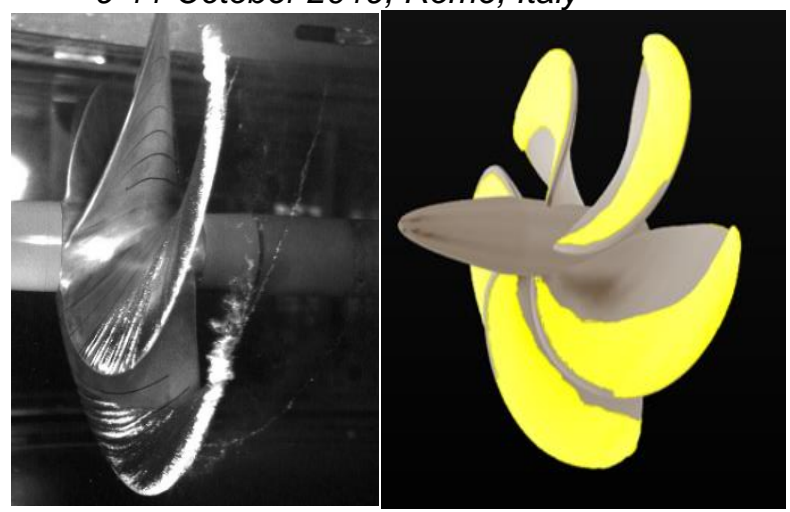

$\mathrm{C} 3, \mathrm{~J}=0.4, \sigma_{\mathrm{N}}=0.72$ (Left; Experiment, Right; RANS $\left.\left(\alpha_{\mathrm{V}}=0.5\right)\right)$

Figure 8. Comparison of cavitation observation for three different conditions at $\mathrm{J}=0.4$

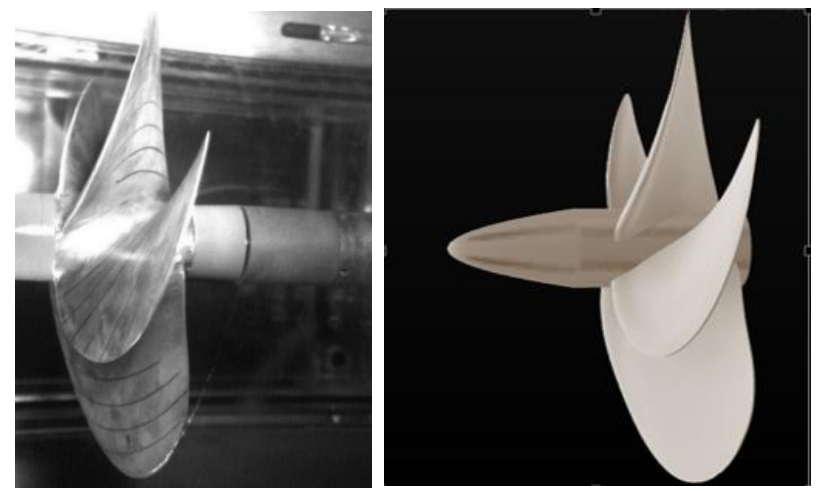

$\mathrm{C} 4, \mathrm{~J}=0.5, \sigma_{N}=3.48\left(\mathrm{Left} ;\right.$ Experiment, Right; RANS $\left.\left(\alpha_{\mathrm{V}}=0.5\right)\right)$
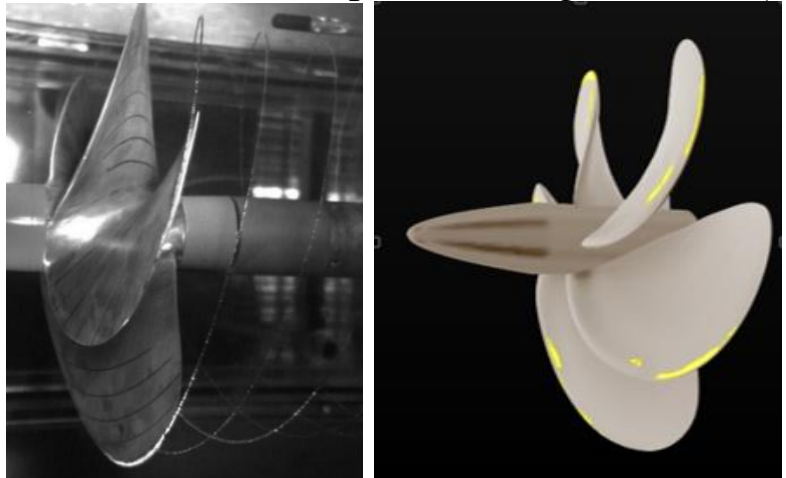

$\mathrm{C} 5, \mathrm{~J}=0.5, \sigma_{N}=2.03$ (Left; Experiment, Right; RANS $\left.\left(\alpha_{\mathrm{V}}=0.5\right)\right)$
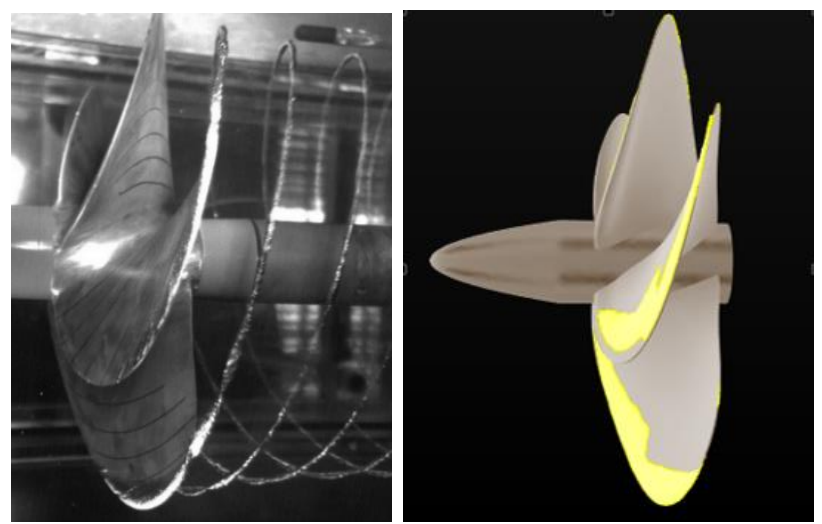

$\mathrm{C} 6, \mathrm{~J}=0.5, \sigma_{\mathrm{N}}=1.13$ (Left; Experiment, Right; RANS $\left.\left(\alpha_{\mathrm{V}}=0.5\right)\right)$

Figure 9. Comparison of cavitation observation for three different conditions at $\mathrm{J}=0.5$ 
Additionally, numerical hydrodynamic thrust coefficients $\left(\mathrm{K}_{\mathrm{T}}\right)$ for different loading conditions were compared with the experiments. Table 4 shows the difference in thrust coefficients between the experimental measurements and numerical calculations. As can be seen in Table 4, thrust coefficients in the numerical calculation were overpredicted in $\mathrm{C} 3$ and $\mathrm{C} 6$ according to experimental results. Therefore, higher thrust value may trigger the blade loading; hence it causes more cavitation area, especially for C3 and C6. On the other hand, apart from the C3 and C6, the numerical calculations presented slightly lower cavitation area; hence the underprediction of the $\mathrm{K}_{\mathrm{T}}$ values in other conditions might be because of this. Besides, it should be noted that tip vortex cavitation might give a contribution to the thrust values in the numerical calculations.

Table 4: Comparison of thrust coefficient $\left(\mathrm{K}_{\mathrm{T}}\right)$ for different loading conditions.

\begin{tabular}{|c|c|c|c|}
\hline & $\mathrm{K}_{\mathrm{T}}(\mathrm{EFD})$ & $\mathrm{K}_{\mathrm{T}}(\mathrm{CFD})$ & Difference (\%) \\
\hline $\mathrm{C} 1$ & 0.244 & 0.233 & -4.5 \\
\hline $\mathrm{C} 2$ & 0.244 & 0.239 & -2.0 \\
\hline $\mathrm{C} 3$ & 0.226 & 0.233 & +3.0 \\
\hline C4 & 0.190 & 0.180 & -5.3 \\
\hline C5 & 0.189 & 0.183 & -3.2 \\
\hline C6 & 0.191 & 0.193 & +1.0 \\
\hline
\end{tabular}

\subsection{Propeller URN Results}

In this section, the results of the cavitation tunnel measurements and numerical prediction for the URN are presented and discussed. In the cavitation tunnel measurements, the corrections, which are explained ANSI/ASA S12.64-2009/Part1, were implemented to determine the net cavitation noise.

Background noise measurements were conducted with a dummy hub model, operating in the same condition as for the propeller. If the difference between the background noise and the propeller noise is smaller than $3 \mathrm{~dB}$, the result is discarded. On the other hand, if the difference is between $3 \mathrm{~dB}$ and $10 \mathrm{~dB}$, the correction is applied with respect to Equation 22. When the difference is more than $10 \mathrm{~dB}$, no correction is included in the calculations (Tani et al. 2017)

$S P L_{N}=10 \log \left[10^{\left(\frac{S P L_{T}}{10}\right)}-10^{\left(\frac{S P L_{B}}{10}\right)}\right]$

where, subscripts N, T and B show net, total and background noise, respectively. As the computed or measured noise levels are dependent on the distance between the noise source and the hydrophone, the distance normalisation is usually implemented as given in Equation 23,

$S P L=S P L_{P}+20 \log \left(\frac{d}{d_{r e f}}\right)$ 
where $S P L_{P}$ is the measured noise level at the specified distance, $\mathrm{dB} ; d$ is the distance between the noise source and hydrophone, $\mathrm{m} ; d_{r e f}$ is the reference distance which is equal to $1 \mathrm{~m}$. The reference distance between the propeller and the hydrophone was kept at H1 (see Figure 2) for the numerical simulations. Later on, numerical results were extrapolated to $1 \mathrm{~m}$ using the ITTC distance normalisation equation. It should be noted that the experimental results were presented as the averaged of spectra of three hydrophones. With the help of the transfer functions, the experimental results were extrapolated to $1 \mathrm{~m}$ distance.

In the numerical URN, in the low-frequency region, predictions were conducted using a hybrid method in which the RANS was equipped with the porous FW-H equation. In the hydrodynamic simulations, the pressure fluctuation induced by the propeller rotation, velocity, and velocity derivatives are taken as inputs to the hydro-acoustic FW-H solver. Thus, FW-H carries the pressures from near field to far-field. Figure 10 and Figure 11 compare the sound pressure levels measured in the cavitation tunnel with the numerical predictions in the low-frequency region. It should be borne in mind that the RANS with FW-H analogy might be used as a suitable tool for prediction of the lowfrequency region, especially to capture the blade harmonics while the RANS approach suffers from accurately capturing the unsteady behaviour of cavitation. Therefore, the broadband noise spectrum, which is strongly affected by the turbulence, can be predicted more precisely using more advanced turbulence modelling such as LES and DES with the FW-H approach to reduce the disadvantage of the RANS approach. Nonetheless, the precise modelling, including the wake effect, should be further investigated.

Figures 10 and 11 compare the numerical and experimental results up to $1 \mathrm{kHz}$ for three different loading conditions. Rather similar trends were observed even if there is a considerable difference for the higher frequency bands. Moreover, 1st Blade Passage Frequency (BPF) values (i.e. $35 * 5=175$ $\mathrm{Hz}$ ) were well captured for all conditions in the numerical simulations while the amplitude of this noise component appeared to be significantly over predicted around 20-25 dB, except for C6. It must be noted that the noise measured at the blade rate frequencies in the experiments may be influenced by the complex acoustical coupling between the propeller and the tunnel test section, which was not taken into account in the current numerical simulations, and neither corrected by the adopted transfer functions. On the other hand, $2^{\text {nd }}$ and $3^{\text {rd }}$ BPF values could not be captured in the numerical predictions. This might be attributed to the unsteady behaviour of the sheet cavitation and/or the grid structure, which triggers the spurious noise induced by the sliding interfaces or inappropriate timestep selection that requires to be investigated by further studies.

* Corresponding author e-mail: savas.sezen@strath.ac.uk 
During the analysis of the experimental results, a prominent peak around $740 \mathrm{~Hz}$ is observed, which is not present in the numerical predictions. This peak is also existed in the background noise measurements, as discussed in (Tani et al. 2017). It is due to the mechanical vibration of one of the two hydrophones supports and expected to be eliminated by the background noise correction. However, a closer investigation of this phenomenon is more complex than the expectation because the intensity of this noise component is higher when the propeller is present since the propeller induced pressure pulses may contribute further into the structural vibration of these supports. Due to the above, this noise component is not relevant when comparing the experimental results with the numerical predictions.
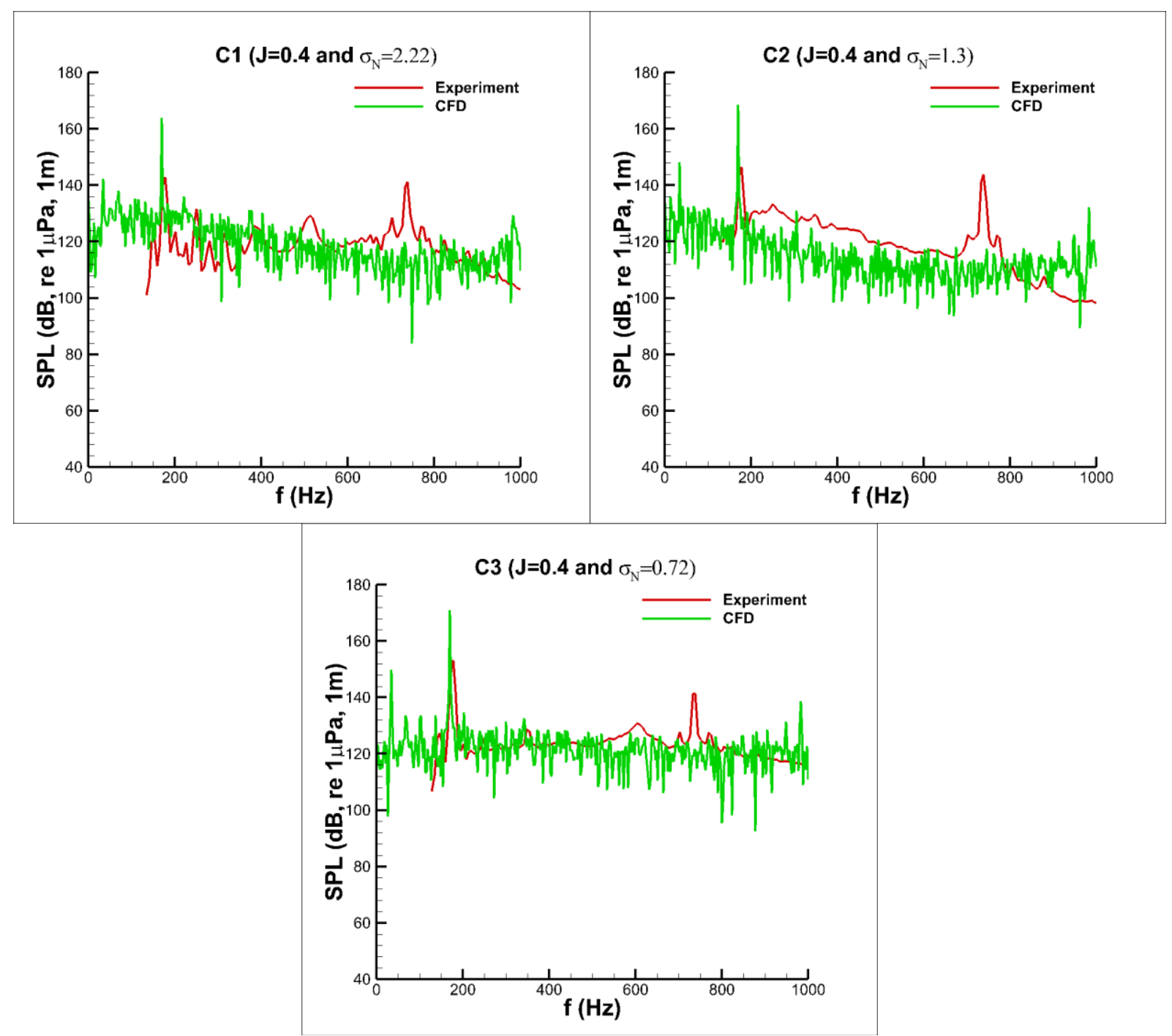

Figure 10. Comparison of experimental and numerical results at $\mathrm{J}=0.4$ for three different loading conditions. 

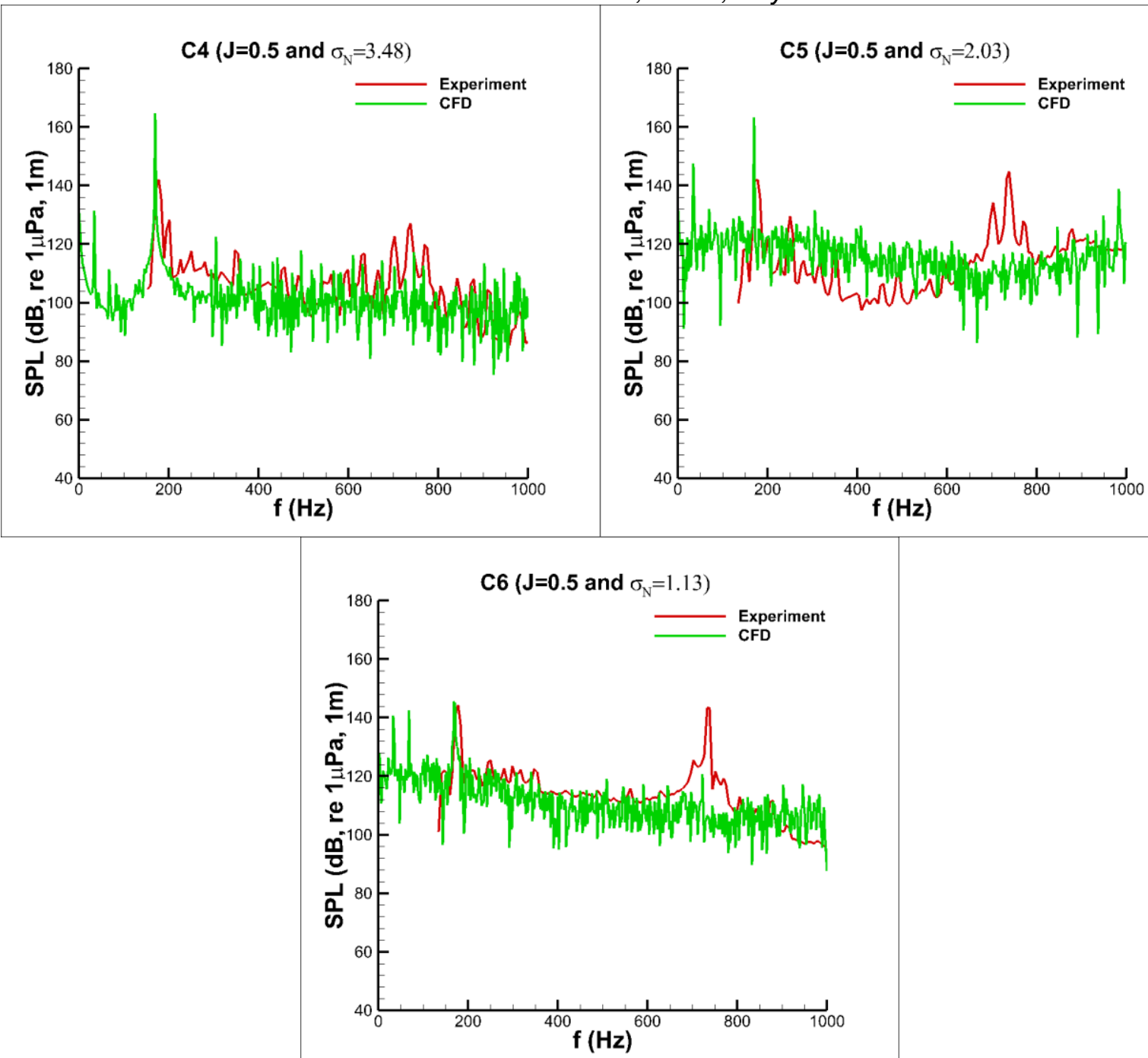

Figure 11. Comparison of experimental and numerical results at $\mathrm{J}=0.5$ for three different loading conditions.

Figure 10 represents the noise levels characterized by the higher loading conditions; around 5-10 dB difference in mid-range levels (between $200-800 \mathrm{~Hz}$ ) was observed for $\mathrm{C} 2$, although $1^{\text {st }}$ blade harmonic was captured in the numerical calculation. It might be since strong tip vortex cavitation was observed during the experiments. The numerical results for $\mathrm{C} 1$ and $\mathrm{C} 3$ show rather good agreement with the experimental results, except the peak around $740 \mathrm{~Hz}$ in the experiment. Similar comments can also be made for other loading conditions in Figure 11. The noise levels measured in the experiments and those predicted by the numerical simulations also indicate similar trends for $\mathrm{C} 4$ and C6 whereas the difference in this trend can be observed in C5 in comparison to other test conditions. As can be seen in Figure 11, the numerical calculation overpredicted the noise levels around 5-10 dB between $250-600 \mathrm{~Hz}$ in C5. Although small underpredictions were observed for C4 and C6 between $200-1000 \mathrm{~Hz}$, the overall trend of the noise spectrums was quite acceptable. Finally, considering the results from the numerical simulations, lower noise levels were observed (around 5-10 dB between 
$200-800 \mathrm{~Hz}$ ), especially for the C2, while the higher noise levels were observed for the C5. However, the characteristic of the spectrums is in a quite good agreement with the experiment. It is worth mentioning that, in the numerical simulations, unexpected pressure oscillations can also be observed due to several issues such as grid structure, time step, the size of the PSD and so on. Additionally, even though the contribution of tip vortex cavitation is considerable for the continuous part of the noise spectrum (broadband), tip vortex cavitation may also have a crucial role in the low-frequency range. Therefore, the difference between numerical results and experiments might be originated from the poor modelling of tip vortex cavitation as well as turbulence. Note that the porous FW-H approach is required to avoid the noise decay, especially in the far-field, as well as including non-linearities into the calculations. On the other hand, the main disadvantage of the porous FW-H is that these surfaces can suffer from the hydrodynamic perturbations (i.e. vortices, shear layers) crossing through the surface and corrupting the signal. Acoustic pressure corruption arises as a result of vortices convected through the permeable surfaces. Thus, it is considered that the selection of an optimal permeable surface is more of an art than a science.

\section{Concluding Remarks}

This study presents the initial results of URN predictions for The Princess Royal model propeller in the uniform flow conditions, and are compared with experimental results from cavitation tunnel tests. In the numerical predictions, first, the hydrodynamic performance of the benchmark model propeller was calculated, and cavitation on the propeller blades was simulated. A verification study was conducted using the GCI technique both for the thrust and torque coefficients for the non-cavitating case. The predicted hydrodynamic characteristics and cavitation pattern on the propeller blades were validated against the experimental results. Following this, the propeller URN was predicted using a hybrid methodology in the low-frequency bands for six different loading conditions. The results of the noise simulations give a rather acceptable prediction with respect to the experimental data in terms of the general shape of the spectrum. Further work is planned, as follows;

- The simulations for broadband noise prediction will be performed using scale resolving methods such as LES and DES for broadband noise prediction.

- Adaptive mesh refinement technique will be implemented on the propeller blade tips to investigate the effects of tip vortex cavitation on the underwater propeller radiated noise.

- Different porous data surfaces will be tested, and the optimum surface will be proposed in order to avoid signal corruption. 
- The experimental set-up will be modelled, including shaft and the numerical results will be compared with those of the current computational domain, which reflects the free field conditions.

- These improvements will be used to investigate model, and full-scale propeller underwater radiated noise under non-uniform flow conditions and in the presence of the hull and rudder.

\section{Acknowledgements}

The first Author is sponsored by Stone Marine Propulsion Ltd of UK and the University of Strathclyde during his PhD study. Also, the granted access to the High-Performance Computing for the West of Scotland (Archie-West) is gratefully acknowledged.

\section{References}

Aktas, B., Atlar, M., Turkmen, S., Shi, W., Sampson, R., Korkut, E., Fitzsimmons, P. (2016), "Propeller cavitation noise investigations of a research vessel using medium size cavitation tunnel tests and fullscale trials," Ocean Eng., vol. 120, pp. 122-135, July.

Bensow, R. and Liefvendahl, M., (2016), An acoustic analogy and scale-resolving flow simulation methodology for the prediction of propeller radiated noise, 31st Symposium on Naval Hydrodynamics, Monterey, California, 11-16 September.

Brentner, K. S. and Farassat, F. (2003), "Modeling aerodynamically generated sound of helicopter rotors," Prog. Aerosp. Sci., vol. 39, no. 2, pp. 83-120, Feb.

Carlton, J. S. (2007), “Marine propellers and propulsion”, 2. ed. Oxford: Butterworth-Heinemann.

Celik, I., Ghia, U., Roache, P.J. and Christopher, J.F. (2008), "Procedure for Estimation and Reporting of Uncertainty Due to Discretization in CFD Applications," J. Fluids Eng., vol. 130, no. 7, p. 078001,

Cianferra, M., Petronio, A. and Armenio, V. (2019), "Non-linear noise from a ship propeller in open sea condition”, Ocean Engineering, 191, 106974.

Di Francescantonio, P. (1997), A new boundary integral formulation for the prediction of sound radiation, vol. 202.

Felli, M., Falchi, M. and Dubbioso, G. (2014), "Direct and indirect experimental methodologies for the hydroacoutic analysis of marine propellers," 30th Symposium on Naval Hydrodynamics, Hobart, Australia.

Gaggero,S. Viviani, M., Tani, G. and Conti, F. (2014), A study on the numerical prediction of propellers cavitating tip vortex, Ocean Engineering, Vol. 92, pp.137-161.

* Corresponding author e-mail: savas.sezen@strath.ac.uk 
Hallander, J., Allenstrom, B., Valdenazzi, F. and Barras, C. (2012), "Predicting Underwater Radiated Noise due to a Cavitating Propeller in a Ship Wake," In Proceedings of the 8th International Symposium on Cavitation (CAV2012), Singapore, pp. 1-7.

Ianniello, S., Muscari, R. and Di Mascio, A. (2013), "Ship underwater noise assessment by the acoustic analogy. Part I: non-linear analysis of a marine propeller in a uniform flow,” J. Mar. Sci. Technol., vol. 18, no. 4, pp. 547-570, Dec.

IMO, (2014), M. R. "Guidelines for the reduction of underwater noise from commercial shipping to address adverse impacts on marine life.

ITTC, 2014. Recommended Procedures and Guidelines, Practical Guidelines for Ship Propulsion CFD, 7.503-033-01.

ITTC, 2014, 27 $7^{\text {th }}$ ITTC Speacialist Committee on Hydrodynamic Noise, Noise Discussion Forms.

Konno, A.. Wakabayashi, K., Yamaguchi, H., Maeda, M., Ishii, N., Soejima, S., Kimura, K. (2002), On the mechanism of the bursting phenomena of propeller tip vortex cavitation, Journal of Marince Science and Technology, 6:181-192.

Kowalczyk, S. and Felicjancik, J. (2016), "Numerical and experimental propeller noise investigations," Ocean Eng., vol. 120, pp. 108-115, July.

Lafeber, F.H., Bosschers, J. and Van Wijngaarden, E. (2015), “Computational and experimental prediction of propeller cavitation noise," OCEANS'15, Genua, Italy.

Li, Da-Qing, Hallander, J. and Johansson. T. (2018), Predicting underwater radiated noise of a full-scale ship with model testing and numerical methods, Ocean Engineering 161,121-135.

Lidtke,A., Lloyd, T., and Vaz, G. (2019), “Acoustic modelling of a propeller subject to non-uniform inflow," Sixth International Symposium on Marine Propulsors, SMP2019, Rome, Italy.

Lighthill, M. J. (1952), “On sound generated aerodynamically I. General theory,” Proc R Soc Lond A, vol. 211, no. 1107, pp. 564-587, March.

Lloyd, T. P., Rijpkema, D. and Wijngaarden, E. (2014), "Implementing the Ffowcs Williams-Hawkings acoustic analogy into a viscous CFD solver,” NUTTS 2014, Gothenburg, Sweden.

Lloyd, T., Rijpkema, D. and Van Wijngaarden, E. (2015), “Marine propeller acoustic modelling: comparing CFD results with an acoustic analogy method," Fourth International Symposium on Marine Propulsors, spm'15, Austin, Texas.

Lyrintzis, S. Anastasios (2003), Surface integral methods in computational aeroacoustics-From the (CFD) near-field to the (Acoustic) far field*, International Journal of Aeroacoustics, Vol:2, pg: 95-128

* Corresponding author e-mail: savas.sezen@strath.ac.uk 
Nitzkorski, Lloyd Zane (2015), A novel porous Ffowcs-Williams and Hawkings acoustic methodology for complex geometries, PhD Thesis, The Faculty of the Graduate School of the University of the Minnesota, USA.

Oshima, A. (1994), "Scaling of Tip Vortex Cavitation Noise of Propeller," Mitsubishi Heavy Industries, Ltd. Technical Review, No. 31.

Richardson, L. F. (1911), "IX. The approximate arithmetical solution by finite differences of physical problems involving differential equations, with an application to the stresses in a masonry dam," Phil Trans R Soc Lond A, vol. 210, no. 459-470, pp. 307-357, Jan..

Sakamoto, N. and Kamiirisa, H. (2018), "Prediction of near field propeller cavitation noise by viscous CFD with semi-empirical approach and its validation in model and full scale," Ocean Eng., vol. 168, pp. 4159, Nov.

Sezen, S. and Kinaci, O.K. (2019), "Incompressible flow assumption in hydro-acoustic predictions of marine propellers," Ocean Eng., vol. 186, August.

Star-CCM+ User Guide, 2018.

Stern, F., Wilson, R. and Shao, J. (2006), "Quantitative V\&V of CFD simulations and certification of CFD codes,” Int. J. Numer. Methods Fluids, vol. 50, no. 11, pp. 1335-1355, April.

Tani, G., Aktas, B., Viviani, M. and Atlar, M. (2017), "Two medium size cavitation tunnel hydro-acoustic benchmark experiment comparisons as part of a round robin test campaign," Ocean Eng., vol. 138, pp. 179-207, July.

Tani, G., Aktas, B., Viviani, M., Yilmaz, N., Miglianti, F., Ferrando, M., Atlar, M. (2019a) "Cavitation tunnel tests for 'The Princess Royal' model propeller behind a 2-dimensional wake screen," Ocean Eng., vol. 172, pp. 829-843, Jan.

Tani, G., Viviani, M., Felli, M., Lafeber, F., Lloyd, T., Aktas, B., Atlar, M., Seol, H., Hallander, J., Sakamoto, N., Kamiirisa, N. (2019b), "Round Robin Test on Radiated Noise of a Cavitating Propeller," Sixth International Symposium on Marine Propulsors, SMP2019, Rome, Italy.

Van Wijngaarden, E. (2005), "Recent developments in predicting propeller-induced hull pressure pulses," $1^{\text {st }}$ International Ship Noise and Vibration, pp. 1-8.

Williams, J. E. F. and Hawkings, D. L. (1969), "Sound generation by turbulence and surfaces in arbitrary motion,” Phil Trans R Soc Lond A, vol. 264, no. 1151, pp. 321-342, May.

Yilmaz, N., Atlar, M., Khorasanchi, M. (2019), An improved Mesh Adaption and Refinement approach to Cavitation Simulation (MARCS) of propellers, Ocean Engineering, Volume 171, January, Pages 139150.

* Corresponding author e-mail: savas.sezen@strath.ac.uk 